\title{
Efecto de la temperatura y concentración en las propiedades reológicas del zumo de sandia (Citrullus lanatus).
}

\section{Purpose of heat and concentration in water melon JUICE (Citrullus lanatus) reological properties.}

Edith A., Vilal, José L., Ninamango'y Lidia, Berrocal"

(1) Facultad de Ciencias Agrarias - Satipo, Universidad Nacional del Centro del Perú Email: edithv3@msn.com

\section{Resumen}

La sandía (Citrullus lanatus L.) es una fruta exótica, de gran consumo por su sabor y aroma agradable. Sin embargo es una fruta que no ha sido ampliamente utilizada para el procesamiento industrial. En este trabajo se determinó el comportamiento reológico del zumo de sandía en el intervalo de $20^{\circ} \mathrm{C}$ a $50^{\circ} \mathrm{C}$ y $7^{\circ}$ Brix a $18^{\circ}$ Brix. El zumo de sandía fue obtenida mediante extracción con tamiz $N^{a}$ 0.5. Las medidas reológicas fueron determinadas en un viscosímetro Brookfield modelo DV-III Plus. El zumo de sandía fue descrita adecuadamente por el modelo Ostwald de Waele, exhibiendo un comportamiento pseudoplástico; al incrementar la temperatura el índice de consistencia ( $k$ ) disminuye y el índice de comportamiento al flujo (n) aumenta, adecuándose a la ecuación de Arrhenius; además al incrementar la concentración el índice de consistencia (k) disminuye y el índice de comportamiento al flujo (n) aumenta.

Palabras clave: zumo de sandía, reología, pseudoplástico

\begin{abstract}
Water melon (Citrullus lanatus L.) is an exotic fruit, which is very consumed for its nice taste and appetizing. However, it is a fruit that has not been widely used in industrial processing. In this work, determine predictive model on the rheological behaviour of water melon juice in the interval from $20^{\circ} \mathrm{C}$ to $50^{\circ} \mathrm{C}$ and $7^{\circ}$ Brix a $18^{\circ}$ Brix was determined. The water melon juice was obtained through net refinery $0.5 \mathrm{~mm}$ opening; they were packed into hermitic bags and subsequently refrigerated to $4^{\circ} \mathrm{C}$. The rheological measurements were determined in a DV-III Plus model Brookfield viscometer. The medlar juice was adequately described by the power law or Ostwald De Waele. For Ostwald De Waele model the temperature and concentration effect were significantly in flow behaviour index (n), and the consistency index (k).
\end{abstract}

Keywords: water melon juice, rheology, pseudo plastic 


\section{Introducción}

Los modelos de comportamiento reológico han sido considerados como una herramienta analítica para proveer información fundamental de la estructura de los alimentos y juega un papel importante en el comportamiento de los flujos. Los modelos matemáticos son aplicados para describir dicho comportamiento, como el modelo matemático de Ostwald de Waele o ley de potencia el cual es utilizado para la caracterización reológica debido a su gran aplicabilidad, el cual ha sido empleado en la investigación para describir el modelo de comportamiento reológico del zumo de sandía (Citrullus lanatus L.).

La reología de las pulpas y jugos tiene una gran importancia en la industria del procesamiento de frutas, incluyendo aplicaciones tecnológicas, sensoriales y de ingeniería, como, por ejemplo, proyectos de bombas, tuberías, intercambiadores de calor y tanques de mezclado (Muller, 1977; Doran, 1999 y Ahmed et al (b), 2007). Estos estudios han sido considerados como una herramienta analítica para proveer información fundamental de la estructura de los alimentos y juega un papel importante en la transferencia de calor a los flujos. Las propiedades reológicas de los alimentos están fuertemente influenciadas por la temperatura, concentración y estado físico de dispersión. Siendo este el caso del zumo de sandía, se determinó el comportamiento reológico del zumo de sandía para el intervalo de experimentación que fue de $20^{\circ} \mathrm{C}$ a $50^{\circ} \mathrm{C}$ para la temperatura; y de $7^{\circ} \mathrm{Brix}$ a $18^{\circ}$ Brix para la concentración, utilizando el viscosímetro Brookfield DV III Plus, con spindle N².

La metodología empleada es la de Mitschka utilizado por Acosta, 2009, la cual contempla la determinación del esfuerzo cortante (t) y la velocidad de corte (y), a partir de los cuales se puede determinar el índice de consistencia (k) y el índice de comportamiento al flujo (n).

Las propiedades reológicas de los jugos de fruta dependen de su variedad, estado de madurez, concentración de jugo y variación de temperatura; los valores de índice de comportamiento al flujo e índice de consistencia son limitados, realizando medidas reológicas en un viscosímetro Brookfield modelo DV-II+ Pro, utilizando la aguja $N^{\circ} 5$ el comportamiento reológico de la pulpa de guayaba se ajusta adecuadamente al modelo de Oswald de Waele o ley de potencia (R2 $\geq$ 0.989), comportándose como un fluido seudoplástico (n $\leq 0.307$ ) y presentando tixotropía las variedades Puerto Rico (14.16\%), Red (I 8.54\%) y DI4 (I 8.48\%). (Andrade, Torres, Montes, Perez y Restan, 2009).

Para un sistema disperso, como es el caso de derivados líquidos de frutas, que fluye con característica no newtoniana el cambio estructural es inmediato y no detectable. Muchos alimentos incluyen en su composición una matriz sólida más o menos elástica y una fase líquida que aporta un elemento viscoso a la estructura total. Estos alimentos presentan un comportamiento reológico que combina características propias de los sólidos elásticos y de los líquidos. (Ramos e lbarz, 2006). La reología de las pulpas y jugos tiene una gran importancia en la industria del procesamiento de frutas, incluyendo aplicaciones tecnológicas, sensoriales y de ingeniería, como, por ejemplo, proyectos de bombas, tuberías, intercambiadores de calor y tanques de mezclado (Torralles, Vendruscolo J y Vendruscolo C., 2006). Estos estudios han sido considerados como una herramienta analítica para proveer información fundamental de la estructura de los alimentos y juega un papel importante en la transferencia de calor a los flujos. Las propiedades reológicas de los alimentos están fuertemente influenciadas por la temperatura, concentración y estado físico de dispersión (Ahmed (a) et al., 2007).

La temperatura tiene una gran influencia en las propiedades físicas de los alimentos, y en concreto, en las propiedades reológicas. Estos parámetros son de especial importancia en las líneas de procesado, condicionando la circulación por diversos equipos y, deben tenerse en cuenta al aplicar tratamientos térmicos de conservación. Además los parámetros reológicos contribuyen a la percepción sensorial por parte del consumidor (Sánchez, Oria y Sánchez- Gimeno, 2007).

El modelo matemático más utilizado para la caracterización reológica es el de la ley de potencia debido a su gran aplicabilidad, donde se relaciona la fuerza de cizallamiento con la variación del gradiente de cizalla (Torralles et al., 2006). La mayoría de las dispersiones en alimentos son sistemas muy complejos y por lo tanto resulta casi imposible alcanzar un claro entendimiento de los mecanismos de flujo que gobiernan sus comportamientos reológicos por una ruta directa; el fluido presento buen ajuste a la ley de la potencia, evidenciando que al incrementar la temperatura el índice de consistencia disminuye mientras el índice de comportamiento al flujo aumenta (Martínez, 2002).

La viscosidad o consistencia de los zumos y purés de frutas es una característica física importante, porque influye en el desarrollo del proceso de elaboración y aceptación del producto por el consumidor (Costell y Durán, 1982 citado por Alvarado, 1993; Velásquez, Monroy y Cortés, 2008). Los datos de la viscosidad pueden emplearse en el diseño de equipos y como medida de control ya que constituyen índices de calidad para muchos productos naturales y elaborados, aunque los datos publicados para jugos son limitados (Alvarado, 1993).

En el rango de temperatura de $5^{\circ} \mathrm{C}$ a $60^{\circ} \mathrm{C}$ y concentraciones de $11^{\circ}$ Brix a $29^{\circ}$ Brix, se demostró que el cremogenado de melocotón tiene comportamiento de fluido pseudoplástico, el cual fue descrito por la ley de la potencia o modelo de Ostwald De Waele. Donde el índice de comportamiento al flujo tiende a aumentar conforme aumenta la temperatura; observándose también el incremento de la energía de activación conforme aumenta el contenido de sólidos solubles, atribuyéndole al contenido en fibra y pectinas; el efecto de la concentración en el cremogenado de melocotón fue descrito 
mediante el modelo tipo exponencial (Garza, 200I).

Existen diversos estudios donde se correlaciona el efecto de la temperatura y la concentración de sólidos solubles en la viscosidad de productos derivados de fruta, esto debido a las diferencias que existe en la composición y estructura de estos productos, no se ha establecido relación alguna entre la energía de activación y la concentración de sólidos solubles (Rao y Anantheswaram., 1984).

Utilizando un Viscosímetro rotacional Brookfield RV-DVII- VG3, spindle $N^{\circ} 2$ se midió las propiedades reológicas del zumo concentrado de naranja valencia a temperaturas de 20 y $30^{\circ} \mathrm{C}$; a concentraciones de 9,5 y $18^{\circ}$ Brix; donde se demostró que el flujo fue No-Newtoniano tipo pseudoplástico que siguió el modelo de Ostwald-De Waale, conocido como la ley de potencia; obteniéndose un índice de comportamiento al flujo (n) de 0,2842 y 0,2584 respectivamente y un índice de consistencia $(k)$ de I,7538 y I,5। 56 en (Pa-s). Para ( $18^{\circ}$ Brix) presentó un índice de comportamiento al flujo (n) de 0,1921 y 0,1919 respectivamente y un índice de consistencia (k) de 2,2623 y 2,0582 (Pa-s); por lo cual se concluye que el índice de comportamiento al flujo (n) disminuye cuando se incrementa la concentración y la temperatura. El índice de consistencia (K) disminuye al incrementarse la temperatura y aumenta al incrementarse la concentración (Acosta y Castro, 1995).

Al realizarse estudios de la influencia de la temperatura y concentración en las características reológicas de la pulpa de carambola (Averrhoa carambola), se utilizó el método de conversión de Mitschka, donde se encontró que el índice de consistencia aumenta al incrementar la concentración, mientras que el índice de comportamiento al flujo disminuye, así mismo el índice de consistencia disminuye al incrementar la temperatura y el índice de comportamiento al flujo aumenta; también se evaluó el efecto de la temperatura con el índice de consistencia encontrando una relación directa de la energía de activación con la concentración de sólidos solubles. El comportamiento reológico al rango de temperaturas y concentraciones es considerado como pseudoplástico (Acosta, 2009).

La sandía (Citrullus lanatus L), es una fruta ampliamente conocida como fruto exótico y apetitoso, posee bajas calorías (Blanco-Blasco, 2003; Alvarado y Blanco 2008). A partir del zumo se pueden obtener aguas saborizadas, bases para vinos, helados y/o añadirse a panes y tortas (Coinsa, 20l I).

Se determinó que en el modelo de Ostwald de Waele al incrementar la temperatura el índice de consistencia ( $k$ ) disminuye y el índice de comportamiento al flujo ( $n$ ) aumenta, mientras que a una misma temperatura al incrementar la concentración el índice de consistencia (k) disminuye y el índice de comportamiento al flujo (n) aumenta. Generándose en la investigación realizada los parámetros de índice de consistencia (k), índice de comportamiento al flujo (n).

Siendo los objetivos planteados:
Determinar el efecto de la temperatura y concentración en las propiedades reológicas del zumo de sandía Citrullus lanantus.

- Determinar la influencia de la temperatura en las propiedades reológicas del zumo de sandía

- Determinar la influencia de la concentración en las propiedades reológicas del zumo de sandía

\section{Materiales y métodos}

Existen investigaciones en reología que aplican el modelo de Ostwald De Waele para caracterizar el comportamiento reológico.

Por lo cual se hace imprescindible tener un rango de temperatura y concentración en las cuales experimentar.

Las propiedades reológicas se definen a partir de la relación existente entre fuerza o sistema de fuerzas externas y su respuesta, ya sea como deformación o flujo. Todo fluido se va a deformar en mayor o menor medida al someterse a un sistema de fuerzas externas. Dicho sistema de fuerzas se representan matemáticamente mediante el esfuerzo cortante o velocidad de flujo de cantidad de movimiento $(\mathrm{t})$, mientras que la respuesta dinámica del fluido se cuantifica mediante la velocidad o tasa de deformación o de cizallamiento (y) (Pierce, 1982).

Se define el esfuerzo de corte o cizalla (t) como la fuerza por unidad de área necesaria para alcanzar una deformación dada. La unidad de esta magnitud más comúnmente utilizada es el Pascal (Pa) (Woyzechowsky, 2002).

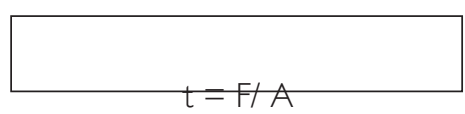

Donde t es el esfuerzo de corte (Pa), F es la fuerza ( $\mathrm{N}$ ) y A es el área (m2).

Por otra parte típicamente hablando en flujos de fluidos, el significado de $t$ equivale a la velocidad de flujo de cantidad de movimiento por unidad de área (mv/A) la cual tiene unidades de (N/m2) (Woyzechowsky, 2002).

El esfuerzo cortante provoca el desplazamiento ordenado de los elementos del fluido, que alcanzan unas velocidades relativas estacionarias que denotaremos $V(x)$. La velocidad de corte se define como el gradiente (velocidad espacial de cambio) del perfil de velocidades. La velocidad de corte es igual a, la diferencia de velocidades en un determinado espacio (Orrego, 2003).

El modelo de ley de potencia de Ostwald de Waele puede modelar fluidos seudoplásticos cuando el exponente, que caracteriza la ley, es mayor que uno. (Sánchez, 2002)

Ecuación: $o=k y$

Siendo:

$\mathrm{o}=$ Esfuerzo cortante. $(\mathrm{Pa})$

$y=$ Velocidad de corte. (I/s) 
$\mathrm{K}=$ Índice de consistencia. (mPan)

$\mathrm{n}=$ Índice de comportamiento de flujo (Adimensional)

El efecto 'de la temperatura sobre el índice de consistencia, se puede describir utilizando la ecuación de Arrhenius mencionado por (Ibarz, 2000).

Siendo: $\quad k=n * \exp (E a / R T)$

$\mathrm{K}=$ Índice de consistencia. (mPan)

$\mathrm{n}=$ Índice de comportamiento de flujo. (Adimensional)

Ea = Energía de activación. $(\mathrm{K} / / \mathrm{mol})$

$\mathrm{R}=$ Constante Universal de los gases. (8.3 I 4 J/g.mol.K)

$\mathrm{T}=$ Temperatura absoluta. ( $\left.{ }^{\circ} \mathrm{K}\right)$

Se han descrito dos tipos de correlaciones o modelos matemáticos para considerar el efecto de la concentración en la viscosidad o índice de consistencia, según los modelos potencial y exponencial expuestos en la ecuación 3 y 4 (Ibarz, 2000).

$\mathrm{n}=\mathrm{k} \mid \mathrm{C}$

$n=k 2 \exp (A 2 C)$

\section{Tipo de investigación}

La investigación es del tipo cuantitativo (Hernández; Fernández y Baptista, 20I0) porque los resultados del comportamiento reológico serán medidos en escala numérica.

\section{Identificación de variables}

a. Variables independientes:

- Temperatura $\left(20^{\circ}, 30^{\circ}, 40^{\circ}\right.$ y $\left.50^{\circ} \mathrm{C}\right)$

- $\quad$ Concentración $\left(7^{\circ}, 9^{\circ}, 12^{\circ}\right.$ y $15^{\circ}$ brix $)$

b. Variables dependientes:

- Índice de consistencia

- $\quad$ Índice de comportamiento al flujo

\section{Diseño de la investigación}

El Modelo estadístico exponencial:

Yij:Eij + KXijn

Donde:

Yij : Esfuerzo cortante

Eij : Error experimental.

K : Índice de consistencia.

Xij : Velocidad de corte.

n : Índice de tendencia al flujo.

\section{Población y muestra}

\section{A. Población}

La población estará constituida por zumo de sandía en estado maduro del distrito de Rio Negro, provincia Satipo.

\section{B. Muestra}

La muestra de la unidad experimental estará constituida por $600 \mathrm{~g}$ de zumo de sandia

\section{Técnicas e instrumentos de recolección de datos}

a) Caracterización Fisicoquímica.

La caracterización de los análisis fisicoquímicos se pueden utilizar diversos métodos: $\mathrm{pH}$ por el método potenciométrico (AOAC - 981.12), sólidos solubles por el método refractométrico (AOAC - 932.12) (2000), acidez total por el método de titulación AOAC -947.05) (2000).

b) Determinación de Parámetros Reológicos. Las medidas reológicas se realizaran mediante un reómetro rotacional Brookfield DV-III+ PRO. (Lewis 1993; Alvarado y Aguilera 200 I).

A partir de los datos de porcentaje de corte y velocidad de rotación del spindle se calculara la tensión cizalla y el gradiente de velocidad mediante la metodología de Mitschka (1982).

\section{Resultados}

\section{Caracterización fisicoquímica del zumo de sandía}

En la tabla I se muestra la caracterización fisicoquímica de sandía en estado maduro en base a $100 \mathrm{~g}$ de porción comestible.

TABLA I Caracterización fisicoquímicos del zumo de sandía

$\begin{array}{lc}\text { CONTROLES } & \text { RESULTADO } \\ \mathrm{pH} & 5,4 \\ \text { Acidez titulable (\% acidez cítrica) } & 23 \\ \text { Sólidos solubles ( }{ }^{\circ} \text { Brix) } & 7 \\ \text { Densidad }(\mathrm{g} / \mathrm{mL}) & 1.005\end{array}$

Fuente: Elaboración propia

De acuerdo a los resultados de la caracterización fisicoquímica de sandía en estado maduro en base a $100 \mathrm{~g}$ de porción comestible; obteniendo el $\mathrm{pH}$ de 5,4. $23 \%$ de acidez, $7^{\circ}$ Brix y I,005 g/ml. de densidad; lo cual se encuentra dentro de los parámetros del codex alimentario, haciéndolo apto para consumo humano.

\section{Parámetros reológicos del zumo de sandía}

Según se observa los valores obtenidos para el coeficiente de determinación R2, tiene valores entre 0,7321 . y 0,9799 . mientras que el coeficiente de correlación (r) resultaron mayores al 89\%, lo que indica una buena relación entre las variables. 
TABLA 2 Valores de las constantes del modelo Ostwald de Waele - ley de la potencia para el zumo de sandia a distintas concentraciones y temperaturas

\begin{tabular}{|c|c|c|c|c|c|}
\hline $\begin{array}{l}\text { Concent } \\
\text { (Brix) }\end{array}$ & $\stackrel{\mathrm{T}}{\mathrm{C}})$ & $\begin{array}{l}\text { Índice de } \\
\text { consist k } \\
\left(\mathrm{Pa} . \mathrm{S}^{\mathrm{n}}\right)\end{array}$ & $\begin{array}{l}\text { flujo }(n) \\
\text { comp al } \\
\text { Indice }\end{array}$ & $\mathrm{R}^{2}$ & $r$ \\
\hline $7^{\circ}$ Brix & $\begin{array}{l}20 \\
30 \\
40 \\
50\end{array}$ & $\begin{array}{l}284,086 \mid \\
\mid 07,6003 \\
94,897 \mid \\
61,7887\end{array}$ & $\begin{array}{l}0,1120 \\
0,1863 \\
0,1934 \\
0,2164\end{array}$ & $\begin{array}{l}0,9751 \\
0,9749 \\
0,87 \mid 4 \\
0,9163\end{array}$ & $\begin{array}{l}0,9804 \\
0,9874 \\
0,9335 \\
0,9572\end{array}$ \\
\hline $9^{\circ}$ Brix & $\begin{array}{l}20 \\
30 \\
40 \\
50\end{array}$ & $\begin{array}{l}185,6650 \\
87,4765 \\
83,6655 \\
47,4299\end{array}$ & $\begin{array}{l}0,1175 \\
0,2172 \\
0,2569 \\
0,2822\end{array}$ & $\begin{array}{l}0,9597 \\
0,9727 \\
0,9031 \\
0,7321\end{array}$ & $\begin{array}{l}0,9796 \\
0,9863 \\
0,9503 \\
0,8556\end{array}$ \\
\hline $12^{\circ}$ Brix & $\begin{array}{l}20 \\
30 \\
40 \\
50\end{array}$ & $\begin{array}{l}\mid 33,6630 \\
85,5992 \\
67,7|5| \\
25,3168\end{array}$ & $\begin{array}{l}0,1724 \\
0,2237 \\
0,2766 \\
0,4114\end{array}$ & $\begin{array}{l}0,9166 \\
0,9351 \\
0,9668 \\
0,9856\end{array}$ & $\begin{array}{l}0,9574 \\
0,9670 \\
0,9833 \\
0,9928\end{array}$ \\
\hline $15^{\circ}$ Brix & $\begin{array}{l}20 \\
30 \\
40 \\
50\end{array}$ & $\begin{array}{l}117,8222 \\
55,8109 \\
53,0181 \\
23,9749\end{array}$ & $\begin{array}{l}0,1735 \\
0,3138 \\
0,3438 \\
0,4511\end{array}$ & $\begin{array}{l}0,9374 \\
0,9799 \\
0,8987 \\
0,7906\end{array}$ & $\begin{array}{l}0,9682 \\
0,9899 \\
0,9480 \\
0,8892\end{array}$ \\
\hline
\end{tabular}

Efecto de la temperatura en las propiedades reológicas del zumo de sandía

a. $\quad$ Efecto de la temperatura sobre el índice de comportamiento al flujo (n) en el zumo de sandía. En la figura I se muestra el efecto de la temperatura en las propiedades reológicas del zumo de sandía.

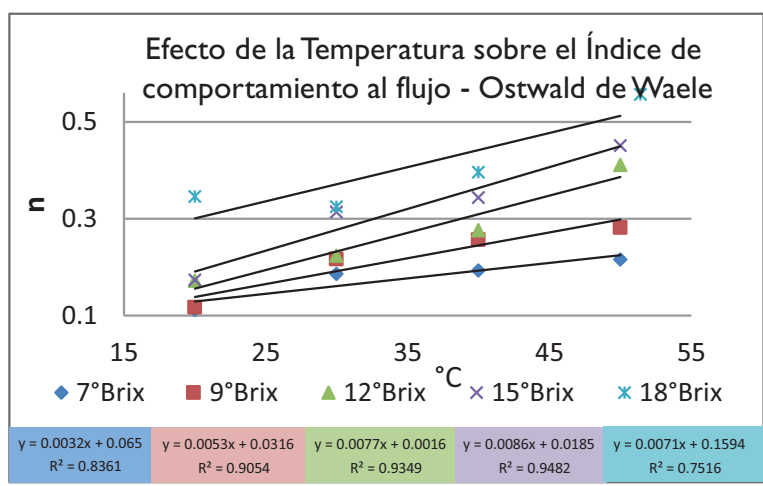

Figura I Efecto de la temperatura sobre el índice de comportamiento al flujo

TABLA 3 Modelos matemáticos del índice de comportamiento al flujo del zumo de sandia en función a la temperatura. Modelo Ostwald deWaele

\begin{tabular}{ccccc}
$\begin{array}{c}\text { Conc } \\
{ }^{\circ} \text { Brix }\end{array}$ & a & B & $\begin{array}{c}\text { Ecuación } \\
y=a+b x\end{array}$ & $R^{2}$ \\
7 & 0.065 & 0.003 & $n=0,065+0,003 *^{\circ} \mathrm{C}$ & 0.836 \\
9 & 0.032 & 0.005 & $n=0,032+0,005{ }^{\circ} \mathrm{C}$ & 0.905 \\
12 & 0.002 & 0.008 & $n=0,002+0,008{ }^{*}{ }^{\circ} \mathrm{C}$ & 0.934 \\
15 & 0.018 & 0.009 & $n=0,018+0,009{ }^{\circ} \mathrm{C}$ & 0.948 \\
18 & 0.159 & 0.007 & $n=0,159+0,007{ }^{*} \mathrm{C}$ & 0.751 \\
\hline
\end{tabular}

Los resultados del coeficiente de determinación, R2 obtenidos están comprendidos desde 0,75। hasta 0,948, lo que indica una asociación directa entre la temperatura y en índice de comportamiento al flujo.

b. Efecto de la temperatura sobre el índice de consistencia $(k)$ en el zumo de sandía

El efecto que ejerce la temperatura sobre el índice de consistencia en el zumo de sandía se midió con la ecuación tipo Arrhenius (Acosta, 2009)

$$
k=\eta_{\infty} \exp \left(\frac{E_{a}}{R T}\right)
$$

TABLA 4 Efecto de la temperatura sobre el índice de consistencia del zumo de sandía a diferente concentración ( ${ }^{\circ}$ Brix), modelo Ostwald de

$\begin{array}{clll}\begin{array}{c}\text { Cont } \\ \left({ }^{\circ} \text { Brix }\right)\end{array} & \begin{array}{l}\text { Ener act } \\ \text { Ea } \\ (\mathrm{KJ} / \mathrm{g} . \mathrm{mol})\end{array} & \begin{array}{l}\text { Facto } r \text { de } \\ \text { frecuencia } \\ \eta_{\infty}\end{array} & R^{2} \\ 7 & 37.265 & 5.446 * 10^{-5} & 0.9009 \\ 9 & 32.650 & 25.623 * 10^{-5} & 0.9152 \\ 12 & 40.830 & 0.777 * 10^{-5} & 0.9072 \\ 15 & 37.953 & 1.934 * 10^{-5} & 0.9165 \\ 18 & 27.914 & 71.163 * 10^{-5} & 0.8788\end{array}$

Los resultados del coeficiente de determinación, $R^{2}$ obtenidos están comprendidos desde 0,8788 hasta 0,9165, lo que indica una asociación directa entre la concentracióm y el índice de consistencia

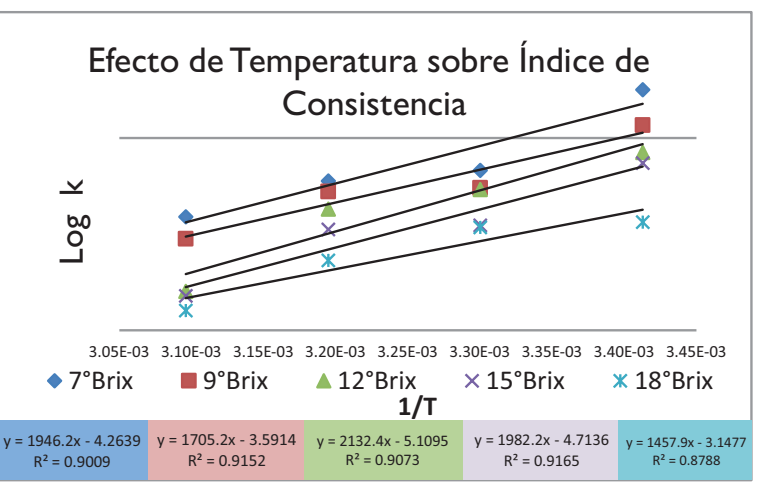

Figura 2 Influencia de la temperatura sobre el índice de consistencia, modelo Ostwald de Waele

\subsection{Efecto de la concentración en las propiedades} reológicas del zumo de sandía

a. $\quad$ Efecto de la concentración sobre el índice de comportamiento al flujo (n) en el zumo de sandía

Los resultados se muestran en la figura 3 y en la tabla 5.

TABLA 5 Modelos matematicos del índice de comportamiento al flujo de zumo de sandía en función a la concentración. Modelo Ostwald deWaele

\begin{tabular}{ccccc}
$\begin{array}{c}\text { Temp } \\
\text { C }\end{array}$ & a & b & \multicolumn{1}{c}{$\begin{array}{c}\text { Ecuación } \\
y=a+b x\end{array}$} & $R^{2}$ \\
20 & $2.404 E-05$ & 0.016 & $n=2,404 \times 10^{-5}+0,012^{*}{ }^{\circ}$ Brix & 0.677 \\
30 & 0.090 & 0.013 & $n=0,090+0,013^{*}{ }^{\circ}$ Brix & 0.914 \\
40 & 0.080 & 0.018 & $n=0,080+0,01$ * $^{\circ}$ Brix & 0.974 \\
50 & 0.014 & 0.030 & $n=0,014+0,03$ ** $^{\circ}$ Brix & 0.979 \\
\hline
\end{tabular}




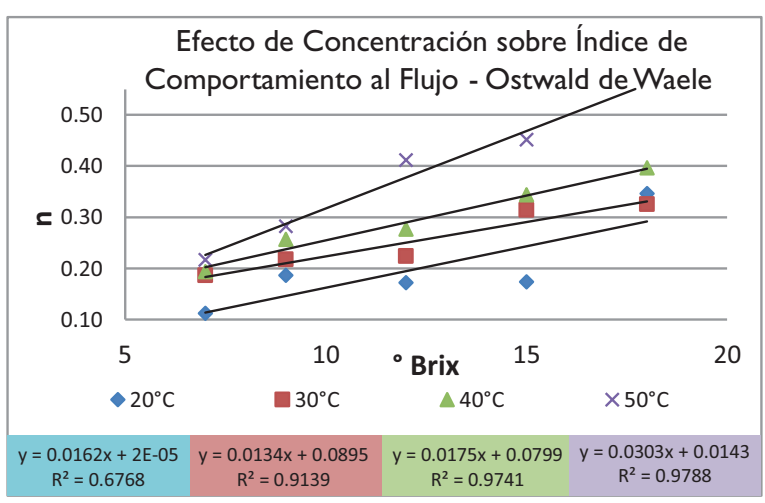

Figura 3 Efecto de la concentración sobreel índice de comportamiento al flujo

Los resultados del coeficiente de determinación, R2 obtenidos están comprendidos desde 0,667 hasta 0,979, lo que indica una asociación directa entre la temperatura y el índice de comportamiento al flujo.

Efecto de la concentración sobre el índice de consistencia ( $k$ ) en el zumo de sandía

Para evaluar el efecto de la concentración sobre el comportamiento reológico del zumo de sandía se utilizó los modelos matemáticos propuestos por (lbartz et al, 2005)

Modelo potencial: $k=K_{-} \mid(C) \wedge\left(A_{-} \mid\right)$

Modelo exponencial: $k=K \_2 \exp \left(A \_2 C\right)$

Dónde: $\mathrm{KI}, \mathrm{K} 2$, A I y A2 son constantes, $\mathrm{C}$ es la concentración en sólidos solubles ( ${ }^{\circ}$ Brix) y $\mathrm{k}$ es el índice de consistencia.

\section{Modelo potencial:}

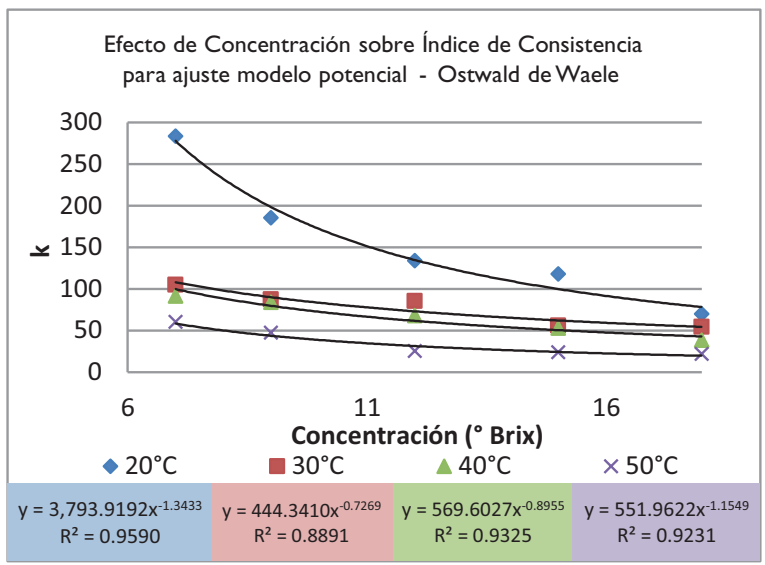

Figura 4 Efecto de la concentración sobre el índice de consistencia para el zumo de sandía. Parámetros resultantes del ajuste al modelo potencial, modelo Ostwald deWaele
TABLA 6 Efecto de la concentración sobre el índice de consistencia del zumo de sandía - modelo potencial, modelo Ostwald deWaele

\begin{tabular}{|c|c|c|c|c|c|}
\hline \multirow{2}{*}{$\begin{array}{c}\text { Temp } \\
\text { C }\end{array}$} & \multirow{2}{*}{$\begin{array}{l}\text { Con } \\
{ }^{\circ} \text { Brix }\end{array}$} & \multirow{2}{*}{$\begin{array}{l}\text { Índice } \\
\text { const (k) } \\
\left(\mathrm{Pa}-\mathrm{S}^{n}\right)\end{array}$} & \multicolumn{3}{|c|}{$\begin{array}{c}\text { Parámetros modelo potencial } \\
k=K_{1}(C)^{A_{1}} \\
\end{array}$} \\
\hline & & & $\mathrm{K}_{1}$ & $A_{1}$ & $\mathrm{R}^{2}$ \\
\hline 20 & $\begin{array}{l}7 \\
9 \\
12 \\
15 \\
18\end{array}$ & $\begin{array}{l}284.086 \\
\mid 85.665 \\
\mid 33.663 \\
|| 7.822 \\
58.10 \mid\end{array}$ & 3793.9192 & -1.3433 & 0.9590 \\
\hline 30 & $\begin{array}{l}7 \\
9 \\
12 \\
15 \\
18\end{array}$ & $\begin{array}{l}107.600 \\
87.476 \\
85.599 \\
55.811 \\
54.462\end{array}$ & 444.3410 & -0.7269 & 0.8891 \\
\hline 40 & $\begin{array}{l}7 \\
9 \\
12 \\
15 \\
18\end{array}$ & $\begin{array}{l}94.897 \\
83.666 \\
67.715 \\
53.018 \\
36.731\end{array}$ & 569.6027 & -0.8955 & 0.9325 \\
\hline 50 & $\begin{array}{l}7 \\
9 \\
12 \\
15 \\
18\end{array}$ & $\begin{array}{l}61.789 \\
47.430 \\
25.317 \\
23.975 \\
20.058\end{array}$ & 551.9622 & -1.1549 & 0.9231 \\
\hline
\end{tabular}

\section{Modelo exponencial:}

TABLA 7 Efecto de la concentración sobre el índice de consistencia del zumo de sandía - modelo exponencial, modelo Ostwald deWaele

\begin{tabular}{|c|c|c|c|c|c|}
\hline \multirow[t]{2}{*}{$\begin{array}{c}\text { Temp } \\
{ }^{\circ} \mathrm{C}\end{array}$} & \multirow[t]{2}{*}{$\begin{array}{l}\text { Conc } \\
{ }^{\circ} \text { Brix }\end{array}$} & \multirow[t]{2}{*}{$\begin{array}{l}\text { Índice de } \\
\text { consis (k) } \\
\left(\mathrm{Pa}-\mathrm{S}^{n}\right)\end{array}$} & \multicolumn{3}{|c|}{$\begin{array}{c}\text { Parámetros modelo } \\
\text { exponencial } \\
k=K_{2} \exp \left(A_{2} C\right) \\
\end{array}$} \\
\hline & & & $K_{2}$ & $A_{2}$ & $\mathrm{R}^{2}$ \\
\hline 20 & $\begin{array}{l}7 \\
9 \\
12 \\
15 \\
18\end{array}$ & $\begin{array}{l}284.086 \\
\mid 85.665 \\
\mid 33.663 \\
\mid 17.822 \\
58.10 \mid\end{array}$ & 578.9944 & -0.1152 & 0.9581 \\
\hline 30 & $\begin{array}{l}7 \\
9 \\
12 \\
15 \\
18\end{array}$ & $\begin{array}{l}107.600 \\
87.476 \\
85.599 \\
55.811 \\
54.462\end{array}$ & 161.7739 & -0.0629 & 0.9044 \\
\hline 40 & $\begin{array}{l}7 \\
9 \\
12 \\
15 \\
18\end{array}$ & $\begin{array}{l}94.897 \\
83.666 \\
67.715 \\
53.018 \\
36.731\end{array}$ & 166.8284 & -0.0788 & 0.9826 \\
\hline 50 & $\begin{array}{l}7 \\
9 \\
12 \\
15 \\
18\end{array}$ & $\begin{array}{l}61.789 \\
47.430 \\
25.317 \\
23.975 \\
20.058\end{array}$ & $\begin{array}{l}105.2 \\
310\end{array}$ & $\begin{array}{l}- \\
0.0 \\
956\end{array}$ & $\begin{array}{l}0.8 \\
605\end{array}$ \\
\hline
\end{tabular}

Para la tabla 7, el coeficiente de determinación R2, para cada temperatura es mayor a 88,91\%; lo cual indica una relación directa entre la concentración y el índice de consistencia para el modelo potencial.

Para la tabla 6, el coeficiente de determinación R2, para cada temperatura es mayor a 86,05\%; lo cual indica una relación directa entre el índice de consistencia y la concentración para el modelo potencial. 


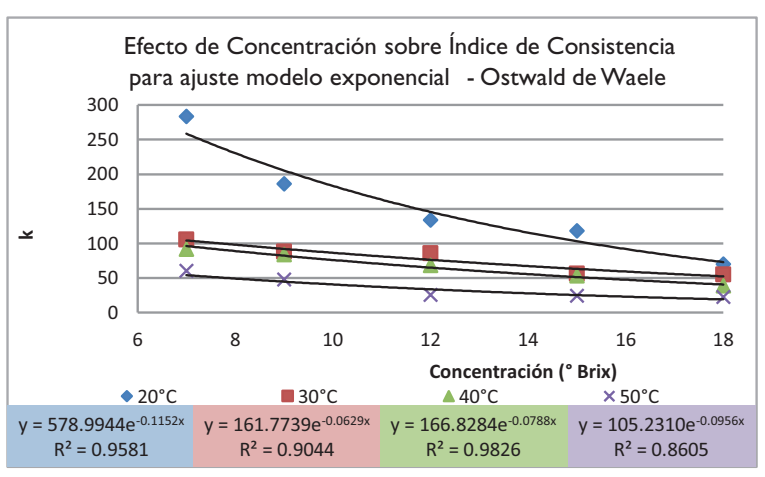

Figura 5 Efecto de la concentración sobre el índice de consistencia para el zumo de sandía. Parámetros resultantes del ajuste al modelo exponencial, modelo Ostwald deWaele

\section{Discusión}

I. De acuerdo al modelo de Ostwald de Waele o ley de la potencia se describe adecuadamente el comportamiento al flujo del zumo de sandía desde un punto de vista práctico se le puede catalogar como un fluido pseudoplástico tal como manifiesta Alvarado (1993) y Acosta (2009). Este modelo ha sido utilizado con éxito para describir el comportamiento reológico del puré de guayaba (Sánchez et al, 2006), en pulpa concentrada de pera (Muñoz et al, 2007), en pulpa de níspero (Andrade et al, 2009) en pulpa de carambola (Acosta, 2009).

2. Para una misma concentración al incrementar la temperatura el índice de consistencia (k) disminuye, lo que concuerda con lo mencionado por Garza (200 I), Acosta y Castro (1998); Graziela et al. (2009) mientras que el índice de comportamiento al flujo (n) aumenta, lo cual concuerda por lo observado por Sánchez, Odria y Sánchez-Gimeno (2007); Acosta (2009); Muñoz et al. (2006); Corso y Sánchez (2008) y el efecto opuesto fue observado por Ponce et al. (2008)

3. A una misma temperatura al incrementar la concentración el índice de consistencia $(k)$ disminuye efecto opuesto al observado por Garza (200I), Muñoz et al. (2008) y el índice de comportamiento al flujo (n) aumenta, lo cual concuerda mencionado por Ponce et al. (2008) mientras que Muñoz et al. (2006) observo comportamiento errático.

\section{Conclusiones}

- $\quad$ Las ecuaciones obtenidas se ajusta bien al modelo de Ostawald De Waele, siendo que para el coeficiente de determinación R2 fueron mayores a 73,2\%, por lo cual el modelo del comportamiento reológico del zumo de sandía se modela mediante la ecuación de Ostwald De Waele o Ley de la Potencia.

- Para una misma concentración el incremento de temperatura da lugar a una disminución en el índice de consistencia; para la muestra de $7^{\circ} \mathrm{Brix}$, el índice de consistencia disminuye desde 284,086। Pa.Sn hasta 61,7887Pa.Sn; para $9^{\circ}$ Brix el índice de consistencia disminuye desde 185,6650Pa.Sn hasta 47.4299Pa.Sn, para $12^{\circ}$ Brix el índice de consistencia disminuye desde 133,6630Pa.Sn hasta 25.3 $168 \mathrm{~Pa}$.Sn, para $15^{\circ}$ Brix el índice de consistencia disminuye desde I 1 7.8222Pa.Sn hasta 23,9749Pa.Sn y para $18^{\circ}$ Brix el índice de consistencia disminuye desde 58, I008Pa.Sn hasta 20,0587Pa.Sn

- Para una misma temperatura al incrementar la concentración ( ${ }^{\circ}$ Brix); a $20^{\circ} \mathrm{C}$ el índice de consistencia disminuye desde 284.086IPa.Sn hasta 58.1008Pa. $\mathrm{Sn}$, a $30^{\circ} \mathrm{C}$ el índice de consistencia disminuye desde 107.6003Pa.Sn hasta $54.4625 \mathrm{~Pa} . \mathrm{Sn}$, a $40^{\circ} \mathrm{Cel}$ índice de consistencia disminuye desde 94.897।Pa.Sn hasta $36.7314 \mathrm{~Pa} . \mathrm{Sn}$ y a $50^{\circ} \mathrm{C}$ el índice de consistencia disminuye desde 61.7887Pa.Sn hasta 20,0587Pa.Sn.

- $\quad$ El zumo de sandía azucarado se comportó como un fluido pseudoplástico para el intervalo de temperatura y concentración estudiado, siendo que la viscosidad aparente disminuye al aumentar la velocidad de deformación.

\section{Referencias}

Acosta y Castro. (1995). Determinación de las características reológicas del zumo concentrado de naranja valencia (Citrus sinensis). Facultad de Ingeniería en Industrias Alimentarias - UNCP. Huancayo - Perú.

Acosta, E R. (2009). Influencia de la temperatura y concentración en las características reológicas de la pulpa CP. Huancayo - Perú.

Ahmed, J. H.; Ramaswamy, L., y Sashidhar, K. (2007). (a) Características reológicas del Tamarindo (Tamarindus indica L.). En: FoodSciTechnol-LEB.40 (2), pp. 225-231

Ahmed, J., H. ramaswamy y K. Sashidhar. (2007). (b) Rheological characteristics of tamarind (Tamarindus indica L.) juice concentrates. Food Sci.

Alvarado J. D. (1993). Viscosidad y energía de activación de jugos filtrados. Revista Española de Ciencia y Tecnología de Alimentos. Número 33 pp87-93.

Alvarado, C.; Blanco, T. (2008). Alimentos Bromatología. Segunda edición. UPC, Lima - Perú.

Andrade R. D., Torres R., Montes E. J., Pérez O. A., Res$\tan$ L. E. y Peña R. E. (b) (2009). Efecto de la temperatura en el comportamiento reológico de la pulpa de níspero (Achras sapota L.). Revista Facultad de Agronomía (LUZ).número 26, Departamento de Ingeniería de alimentos, Facultad de Ciencias Agrícolas, Universidad de Córdoba, Montería, e Ingeniería de alimentos, Facultad de Ciencias Agrícolas, Universidad de Córdoba, Colombia pp 599-612. 
Blanco-Blasco, T. y Alvarado, O. C. (2003). Alimentos bromatología. Fundación Ajinomoto para el desarrollo de la comunidad. Lima - Perú.

CODEX STAN 247-2005. NORMA GENERAL DEL CODEX PARA ZUMOS (JUGOS) Y NÉCTARES DE FRUTAS COINSA - CONCENTRADOS INDUSTRIALES S.A. (20II). Bebidas División Sabores - Jarabe Terminado, Concentrados Industriales S.A disponible en www.e-coinsa@ contal.com.

Corzo O., Sánchez M. (2008). Estudio reológico del proceso de elaboración de aceite de maíz. Departamento de Tecnología de Alimentos. Núcleo de Nueva Esparta. Universidad de Oriente. Revista saber Vol. 20 No 3: 329-333. Boca del Río Venezuela.

Doram P. M. (1999). Principios de ingeniería en los bioprocesos. Editorial Acribia S.A., Zaragoza España.

Garza, Salvador. (200I). Caracterización reológica y microbiológica, y cinéticas de deterioro en cremogenado de melocotón. Servei de Publicacions. Universidad de Lleida. ISBN: 84-89727-64-3.

Graziella-Colato A., Faria F. R.; Takeitii C. Y.; \& Park K. (2009). Rheological behavior of blueberry. Campinas StateUniversity, Brazil.

Hernandez R.; Fernandez C. Y Baptista P. (20 I 0). Metodología de la investigación, México ediciones Mc Graw Hill 4ta ed.

IBARZ. (2000). Métodos Experimentales en la Ingeniería Alimentaria. España: Editorial Acribia. pp. $143-$ 148.

Martínez I. R. M. 2002. Caracterización del comportamiento reológico de sopa crema orientada a control de calidad. Escuela de Ingeniería en Alimentos. Facultad de Ciencias Agrarias universidad Austral de Chile. Valdivia - Chile.

Mischka, P. 1982. Simple Conversion of Brookfield RVT Reading into Viscosity Functions. Rheol.

Muller H. G. 1977. Introducción a la reología de los alimentos. Editorial Acribia, Zaragoza - España.

Muñoz E, Rubio L, Cabeza M, Possa H. 2006. Modelo predictivo del comportamiento reológico de pulpas de pera. Facultad de Ciencias Aplicadas a la Industria, UNCuyo.
Orrego, C. 2003. Procesamiento De Alimentos. Universidad Nacional de Colombia.

Pierce, P. 1982, Rheological Measurements, New Yord: Edición John Wiley \&Sons.

Ramos A. M., Ibarz A. 2006. Comportamiento Viscoelástico de Pulpa de Membrillo en Función de la Concentración de Sólidos Solubles. Departamento de Tecnología de Alimentos. Universidad Federal de Viçosa y Departamento de Tecnología de Alimentos. Universidad de Lleida, España.

Rao, M. A. Y Anantheswaram, R.c. 1984. Rheology of fluids. Food Processing, Vol. 36, p. | | 6- 126.

Sánchez. 2002. Estudio de Fluidos no newtonianos con los métodos de volúmenes y elementos finitos. Revista de Ingeniería. Universidad de Santiago de Chile. 165 p.

Sánchez C., Oria R. Y Sánchez- Gimeno A.c. 2007. Efecto de la temperatura en las propiedades reológicas de purés de guayaba (Psidium guajaba L.) Grupo de Investigación en Alimentos de Origen Vegetal. Universidad de Zaragoza y Unellez. Programa Procesos Industriales. Estado Cojedes. España y Venezuela.

Torralles, R:; Vendruscolo, J. Y Vendruscolo, C. 2006. Reológia de purê homogeneizado de Pêssego: Efeito da temperatura e concentração. Braz. J. FoodTechnol. 9 ( I):-8.

Velásquez H. J. C., Monroy L. F. A. Y Cortés M. E. A. 2008. Estudio Preliminar del Comportamiento Reologico Bajo Compresión Unidireccional de la Pulpa de Plátano (Musa AAB Simmonds) Universidad Nacional de Colombia, Medellín - Colombia.

Woyzechowsky, L. 2002. Estudio de reología y transformación del almidón de maíz en un reómetro capilar. Trabajo de Grado, Universidad Simón Bolívar. 99 p. 